This article was downloaded by: [Research Center of Eco-Environmental Sciences]

On: 13 March 2015, At: 00:04

Publisher: Taylor \& Francis

Informa Ltd Registered in England and Wales Registered Number: 1072954 Registered office: Mortimer House, 37-41 Mortimer Street, London W1T 3J H, UK

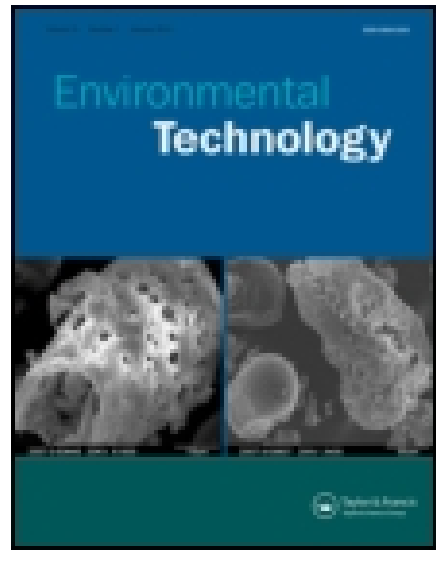

CrossMark

\title{
Environmental Technology
}

Publication details, including instructions for authors and subscription information: http:// www.tandfonline.com/loi/tent20

\section{Research on red mud treatment by a circulating superconducting magnetic separator}

\author{
Yiran $\mathrm{Li}^{\mathrm{ab}}$, Haoshu Chen ${ }^{\mathrm{b}}$, J un Wang ${ }^{\mathrm{c}}$, Fengyu $\mathrm{Xu}^{\mathrm{b}} \&$ Weimin Zhang ${ }^{\mathrm{a}}$ \\ ${ }^{a}$ Department of Water Resources and Environmental Engineering, East China Institute of \\ Technology, Nanchang, 330013 J iangxi, People's Republic of China \\ b J ack_Zhongke Superconducting Technology Co., LTD. , J iangyin, 214404 J iangsu, People's \\ Republic of China \\ ' State Key Laboratory of Environmental Aquatic Chemistry, Research Center for Eco- \\ Environmental Sciences, Chinese Academy of Sciences, P. O. Box 2871, Beij ing 100085, \\ People's Republic of China \\ Accepted author version posted online: 15 Nov 2013. Published online: 17 Dec 2013.
}

Click for updates

To cite this article: Yiran Li, Haoshu Chen, J un Wang, Fengyu Xu \& Weimin Zhang (2014) Research on red mud treatment by a circulating superconducting magnetic separator, Environmental Technology, 35:10, 1243-1249, DOI: $10.1080 / 09593330.2013 .865763$

To link to this article: http:// dx. doi.org/ 10.1080/09593330.2013.865763

\section{PLEASE SCROLL DOWN FOR ARTICLE}

Taylor \& Francis makes every effort to ensure the accuracy of all the information (the "Content") contained in the publications on our platform. However, Taylor \& Francis, our agents, and our licensors make no representations or warranties whatsoever as to the accuracy, completeness, or suitability for any purpose of the Content. Any opinions and views expressed in this publication are the opinions and views of the authors, and are not the views of or endorsed by Taylor \& Francis. The accuracy of the Content should not be relied upon and should be independently verified with primary sources of information. Taylor and Francis shall not be liable for any losses, actions, claims, proceedings, demands, costs, expenses, damages, and other liabilities whatsoever or howsoever caused arising directly or indirectly in connection with, in relation to or arising out of the use of the Content.

This article may be used for research, teaching, and private study purposes. Any substantial or systematic reproduction, redistribution, reselling, loan, sub-licensing, systematic supply, or distribution in any form to anyone is expressly forbidden. Terms \& Conditions of access and use can be found at http:// www.tandfonline.com/page/terms-and-conditions 


\title{
Research on red mud treatment by a circulating superconducting magnetic separator
}

\author{
Yiran $\mathrm{Li}^{\mathrm{a}}$,b*, Haoshu Chen ${ }^{\mathrm{b}}$, Jun Wang ${ }^{\mathrm{c}}$, Fengyu $\mathrm{Xu}^{\mathrm{b}}$ and Weimin Zhang ${ }^{\mathrm{a}}$ \\ ${ }^{a}$ Department of Water Resources and Environmental Engineering, East China Institute of Technology, Nanchang, 330013 Jiangxi, \\ People's Republic of China; ${ }^{b} J a c k$ Zhongke Superconducting Technology Co., LTD., Jiangyin, 214404 Jiangsu, \\ People's Republic of China; ' State Key Laboratory of Environmental Aquatic Chemistry, Research Center for Eco-Environmental \\ Sciences, Chinese Academy of Sciences, P.O. Box 2871, Beijing 100085, People's Republic of China
}

(Received 5 December 2012; accepted 9 November 2013)

\begin{abstract}
Red mud (RM) accumulated over the years and caused a serious environmental problem. Iron-rich fraction separation is a cost-effective way to reduce the amount of disposal RM. A circulating high-gradient superconducting magnetic separator was produced in this work. Steel wool was filled in the circulating boxes. The boxes were connected by two chains, which moved in and out the magnetic field by a drive motor. The efficiency of iron-rich RM separation by the superconducting magnetic separator was investigated. An amount of $25 \%$ (w/w) iron-rich RM fractions with a grade of $65 \%$ were separated from the $56 \%$ iron content raw RM. The parameters of the steel wool matrix were important in controlling the iron-rich RM magnetic separation. Finer steel wool increased the iron recovery ratio, but decreased the grade of the iron-rich RM concentrates. Microscopic photographs of the RM particles showed that opaque mineral particles were enriched in the collected RM. The particle size distributions of raw, concentrate and residue RM were measured. The increased particle size of concentrate RM implied that large particles were entrapped in the steel wool matrix.
\end{abstract}

Keywords: red mud; circulating; superconducting; magnetic separator; iron recovery

\section{Introduction}

Presently, about 120 million tonnes of alumina are produced per year all over the world. Each tonne of alumina will produce an amount of 1-1.5 ton of residues. Bayer red mud (RM) is the residue of the aluminium industry formed during the alkaline extraction of alumina from bauxite by the Bayer process. RM accumulated over the years and caused a serious environmental problem because of its high alkalinity and the large amount.[1]

The utilization of RM is of environment and economic significance. Lots of researches have focused on the reuse of RM, which was used as catalysis reagents, environmental applications, ceramics, etc.[1-3] However, the utilization of RM in industrial application is still a worldwide problem. The emission reductions and costs were two main factors controlling the RM utilization technology. Presently, a large amount of RM with high moisture content were still open storage in the RM yard by building dams.

It was reported that an amount of more than $25 \%$ of iron oxide was contented in the RM. Magnetic separation is a method for the separation of particles based on their magnetic properties. When magnetic force exceeds the drag force and gravity, the particle can be separated. Iron recovery from $\mathrm{RM}$ with the reduction roasting process followed by magnetic separation was widely reported.[4]
Systemic experimental research and theoretical analysis were reported on the recovery of iron from the bauxite residue using the direct reduction roasting process. [5] However, roasting was a high-cost procedure which can hardly be used in the industrial scale.

The traditional magnetic separation process was hard to separate the iron particles less than $100 \mu \mathrm{m}$. Considering the extreme fine particles of RM, an advanced magnetic separation method should be considered.[6] Generally, it is feasible to separate iron-rich RM from the bauxite residues by high-gradient magnetic separation (HGMS).[7] High iron content RM could be directly added in a blast furnace. In the HGMS, the values of the high iron content RM and the corresponding emission reduction should cover the cost of the iron separation process. It was reported that the pulsating HGMS was used in the Shandong Branch, Aluminum Corporation of China.[8]

Superconducting magnets have a number of advantages over resistive electromagnets in fine magnetic materials recovery.[9] First, the superconducting magnets can achieve an order of magnitude stronger field than ordinary ferromagnetic-core electromagnets, which can be more efficient in separating fine magnetic particles. Second, the large magnetic-field region of superconducting magnets was advantaged for industrial application. Third, the power

\footnotetext{
*Corresponding author. Email: lyr2006xd@gmail.com
} 
consumption of superconducting magnets was much lower compared with the traditional electromagnets.

The first high-gradient superconducting magnetic separator for kaolin treatment was produced by Goonvean (CRYOFILTER RHG) in 1989. The separator was equipped with a reciprocating canister, which was capable of producing a $5 \mathrm{~T}$ magnetic field.[10] In decades, superconducting magnetic separator was widely investigated in mineral treatment [11,12] and water treatment.[13] However, there were little works focused on the superconducting magnetic separator used for iron recovery, especially for RM treatment. In this work, a novel high-gradient superconducting magnetic separator with a circulating box system was reported. Meanwhile, the efficiency of iron recovery from RM was also investigated.

\section{Superconducting magnetic separation system}

\subsection{Superconducting magnet}

The room temperature bore of the superconducting magnet is $902 \mathrm{~mm}$. The central magnetic field of the magnet is $1.2 \mathrm{~T}$. The length of the coil is $1460 \mathrm{~mm}$. The work current of the magnet is $167 \mathrm{~A}$. Some parameters of the magnet are shown in Table 1. The simulated distribution of magnetic field is shown in Figure 1.

Magnetic field and the magnetic-field region are the main requirements for magnets in HGMS. The main magnetic mineral composition of RM is hematite, which is fine weakly magnetic mineral. HGMS could provide strong magnetic drag force for separate the iron-rich RM.

\subsection{Magnetic separation system}

The circulating HGMS is based on the superconducting magnet mentioned above. The photograph of the magnetic separator is shown in Figure 2.

As shown in Figure 2(c), a circulating system was inserted in the superconducting magnet. The schematic diagrams of the circulating system with or without superconducting magnet are shown in Figure 2(a) and 2(b). The magnetic field of the feeds inlet was 1.0-1.2 T. Weak magnetic materials were collected in the residues pool. In order

Table 1. Main parameters of the superconducting magnets.

\begin{tabular}{lc}
\hline Parameter & Value \\
\hline Inner diameter (mm) & 902 \\
Outer diameter (mm) & 1820 \\
length of the coil (mm) & 1460 \\
Length (mm) & 1600 \\
Magnetic field (T) & 1.2 \\
Work current (A) & 167 \\
Number of coils & 6 \\
Operating temperature (K) & 4.7 \\
Conductor type Niobium-titaniumalloys \\
\hline
\end{tabular}

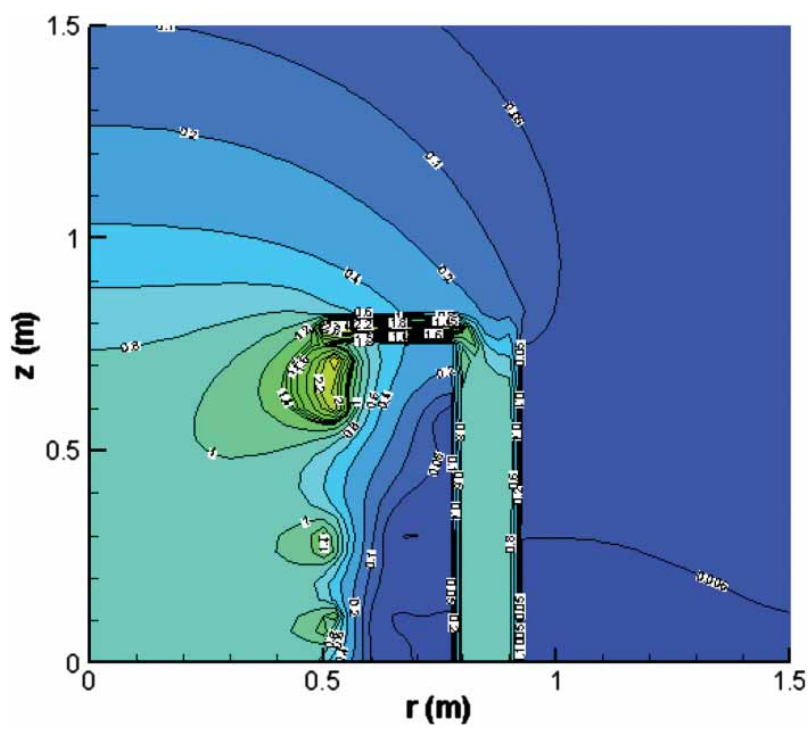

Figure 1. The distribution of simulated magnetic field when the current was $167 \mathrm{~A}$.

to collect the separated iron-rich RM particles, named concentrate RM, two wash points of the magnetic separator were designed out of the magnet, as shown in Figure 2.

The circulating system was consisted of boxes, chains and rotating motor. The boxes continuously rotated along the vertical direction of the ground. Steel wool was filled in the circulating boxes. The photograph of the circulating box is shown in Figure 3(a). The length, broad and deep of the box was 600,100 and $100 \mathrm{~mm}$, respectively. The inlet of the boxes was at the top, surface and the outlet of the boxes was at the side surface. The 2\# 3\# 4\# and 5\# steel wools were provided by Changsha research institute of mining and metallurgy Co. LTD. The height and width of steel wools are shown in Table 2.

An amount of $150-500 \mathrm{~g}$ steel wool, shown in Figure 3(b), was filled in each circulating box. The volume fill rate was about 5\%. As shown in Figure 3(a), steel wool was fixed in the circulating box with a cover, which was produced using stainless steel wire.

\section{Materials and experiments}

\subsection{RM samples}

RM samples were provided by the No.2 factory of Shandong Branch, Aluminum Corporation of China, which were collected in October 2011. The RM samples collected in this work were the overflow fractions after the gravity cyclone separation. The mean particle size of the RM samples was about $10 \mu \mathrm{m}$. The chemical compositions of RM are shown in Table 3 .

\subsection{Procedures}

An amount of $10 \mathrm{~kg}$ RM (dry weight) was homogeneous dispersed in $50 \mathrm{~L}$ water. The RM solutions were pumped 

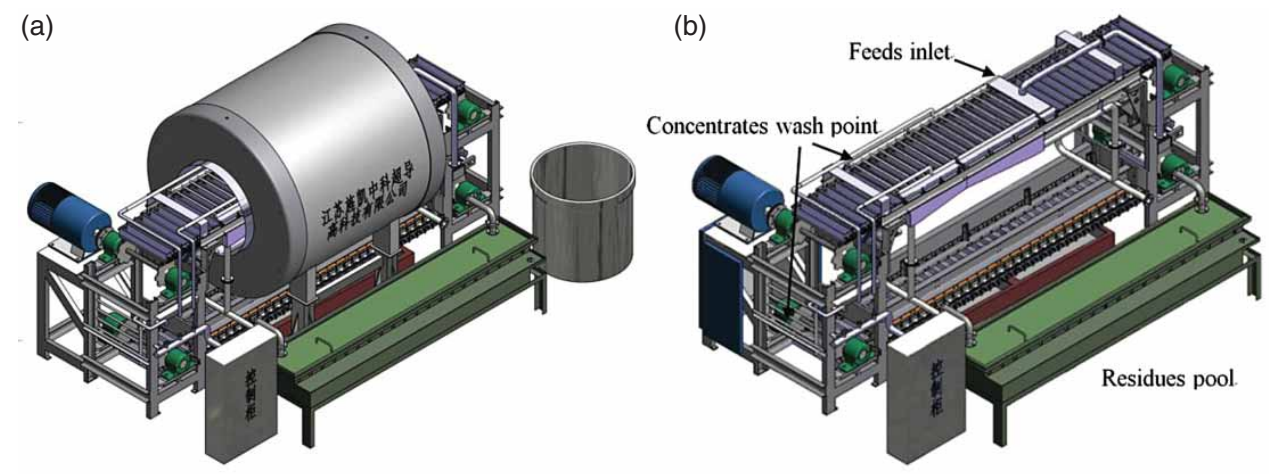

(c)

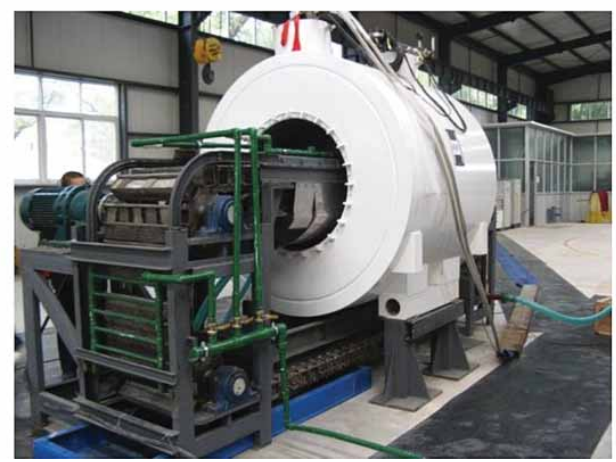

Figure 2. Paragraph of the circulating superconducting magnetic separator.

(a)

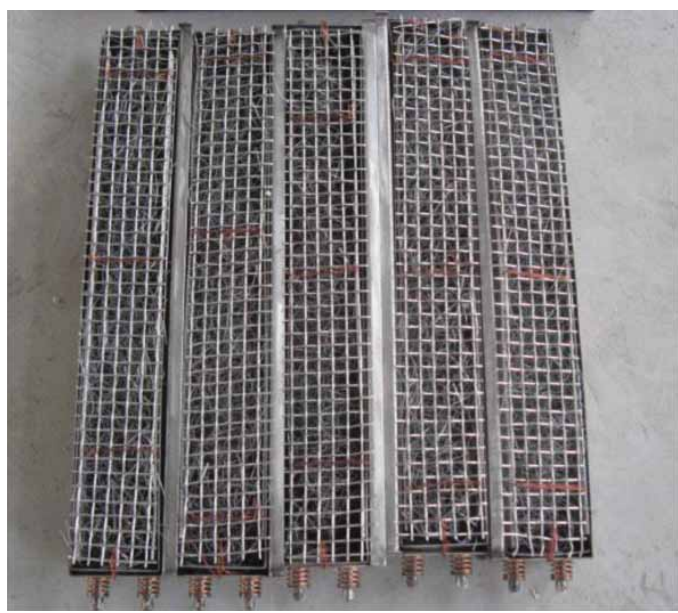

(b)

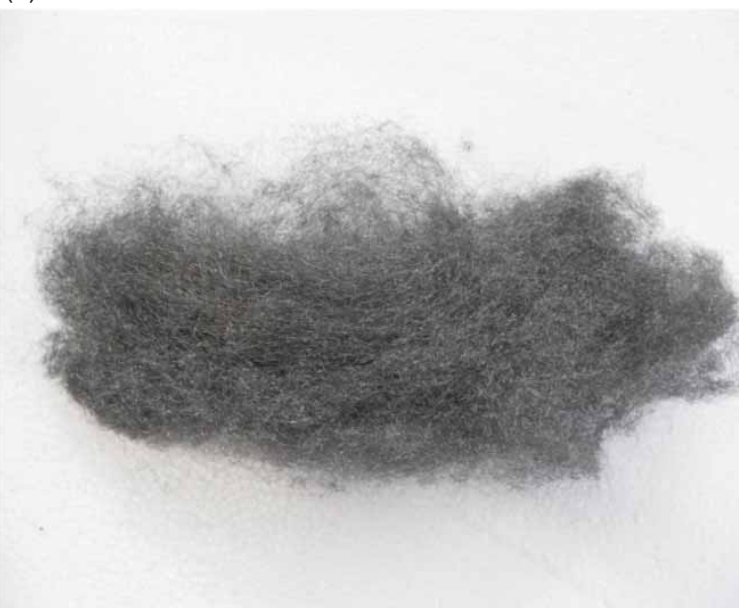

Figure 3. The circulating boxes (a) and the steel wool (b).

Table 2. The width and height parameters of the steel wools.

\begin{tabular}{lcc}
\hline Steel wool & Width $(\mathrm{mm})$ & Height $(\mathrm{mm})$ \\
\hline $2 \#$ & 0.07 & 0.15 \\
$3 \#$ & 0.07 & 0.20 \\
$4 \#$ & 0.12 & 0.20 \\
$5 \#$ & 0.13 & 0.27 \\
\hline
\end{tabular}

into the superconducting magnetic separation system by a diaphragm pump. The flow rate was controlled at 1 ton/h by an electromagnetic flowmeter. The fractions flowed through
Table 3. Chemical compositions of the raw RM.

\begin{tabular}{lllllllll}
\hline$\%$ & $\mathrm{SiO}_{2}$ & $\mathrm{Fe}_{2} \mathrm{O}_{3}$ & $\mathrm{Al}_{2} \mathrm{O}_{3}$ & $\mathrm{CaO}$ & $\mathrm{TiO}_{2}$ & $\mathrm{Na}_{2} \mathrm{O}$ & $\mathrm{K}_{2} \mathrm{O}$ & Ignition loss \\
\hline $\mathrm{RM}$ & 6.62 & 56.14 & 12.81 & 0.98 & 2.40 & 3.18 & 0.05 & 11.65 \\
\hline
\end{tabular}

the steel wool matrix were collected in the residues pool. Meanwhile, the magnetic fractions adsorbed in the steel wool matrix were washed out after the circulating box moved out of the magnets. The concentrates and residues were collected during the magnetic separation process. 
After one cycle of magnetic separation, residues were pumped again into the magnetic separation system. The iron content of the concentrates and the residues was measured. Meanwhile, the mass recoveries of different fractions were also measured.

\subsection{Methods of analysis}

Iron content in the RM was measured by inductively coupled plasma atomic emission spectroscopy (Prodigy) after the solid phase was digested by a microwave oven procedure. The digestion solution was Aqua regia $(3+1$, $\mathrm{HCl}-\mathrm{HNO}_{3}$ ). An amount of $50 \mathrm{~mL}$ samples of the concentrates or residues was collected, and the solid content was measured after drying. Size distributions were determined using a Winner 318 A Split Spraying Laser Particle Size Analyzers (Winner instruments, China) with an ultrasonic deconcentrator, which ascertains size by analysis of forward scattered light. The micrograph and microanalysis of the samples were determined using a Transmission polarizing microscope UP200i (Phtoelectric Technology Co. China). Image-processing software was used to quantitatively analyse the content of opaque particles in RM pictures. An $\mathrm{X}$-ray diffractometer (XRD) (ShimadzuXRD-6000) operated at $40 \mathrm{kV}$ and $30 \mathrm{~mA}$ was used to identify the crystal structure and crystallinity with $\mathrm{Cu} \mathrm{K} \alpha(1=0.15418 \mathrm{~nm})$ radiation over the range of 2 from $2.6^{\circ}$ to $70^{\circ}$. The volumes of the concentrates or residues were measured after the magnetic separation process. Therefore, the mass recoveries of the magnetic separation process were calculated. Experiments of the same conditions were performed in triplicate; the mean values and standard deviation were considered.

\section{Results and discussions}

\subsection{Mineral phase compositions of RM}

The XRD pattern of the RM is shown in Figure 4. Hematite, $\mathrm{Al}(\mathrm{OH})_{3}$ and quartz were examined in this sample. The characterization of RM was widely investigated before.[14] Mineral phase compositions of RM were investigated several times in our previous works.[7] Although the mineral phase compositions of RM show some discrepancies in different batches, hematite, anatase, quartz, $\mathrm{CaCO}_{3}$ and $\alpha-\mathrm{Al}(\mathrm{OH})_{3}$ were frequently detected by XRD.

\subsection{The advantages of the circulating superconducting magnetic separator}

Advantages of the circulating superconducting magnetic separator were discussed as follows. First, the circulating superconducting magnetic separator was effective in power and cool water saving compared with the conventional HGMS. Second, the processing capacity of the circulating superconducting magnetic separator was evaluated. The feed rate could be higher than 70 ton/h; the corresponding rotation speed of the boxes was $2 \mathrm{rpm}$. At this feed rate,

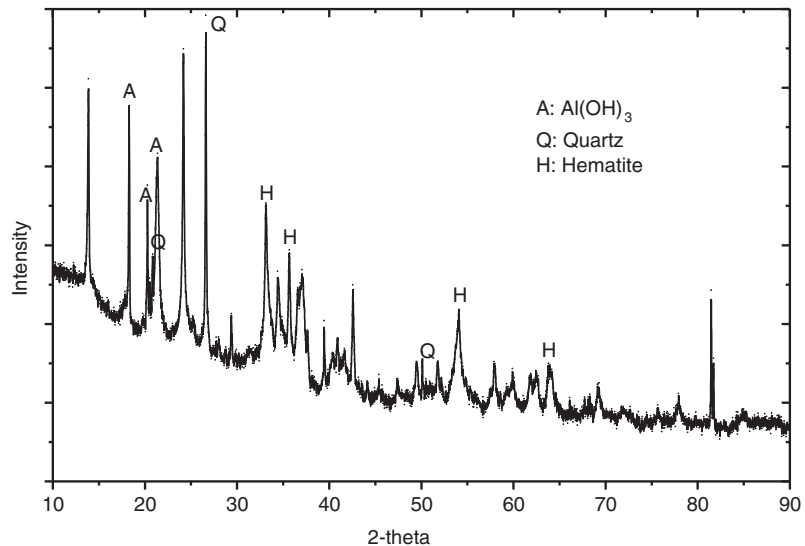

Figure 4. XRD patterns of the RM.

about 14 ton RM in dry weight was treated within $1 \mathrm{~h}$. Moreover, a further improvement in increasing the magnetic field utilization efficiency could provide higher production compared with the traditional HGMS. Third, compared with the reciprocating canister, the circulating boxes facilitated the steel wool installing and dismantling. Meanwhile, there was little water dead ends in the circulating boxes, and which could be thoroughly cleaned separately. The circulating superconducting magnetic separator is a novel attempt for the future HGMS device.

\subsection{Three stages of magnetic separation}

The iron contents of the feeds, concentrates and residues RM of three stages' magnetic separation are shown in Figure 5. The 4\# steel wool was filled in the circulating boxes. During the first stage of magnetic separations, iron oxide content in the RM was improved from $56 \%$ to $65 \%$. There was $730 \mathrm{~g}$ concentrated RM and $220 \mathrm{~g}$ residue RM collected in the stage 1 and the corresponding mass recovery was $95 \%$. Residues in the first stage magnetic separation were pumped into the magnetic separation system for

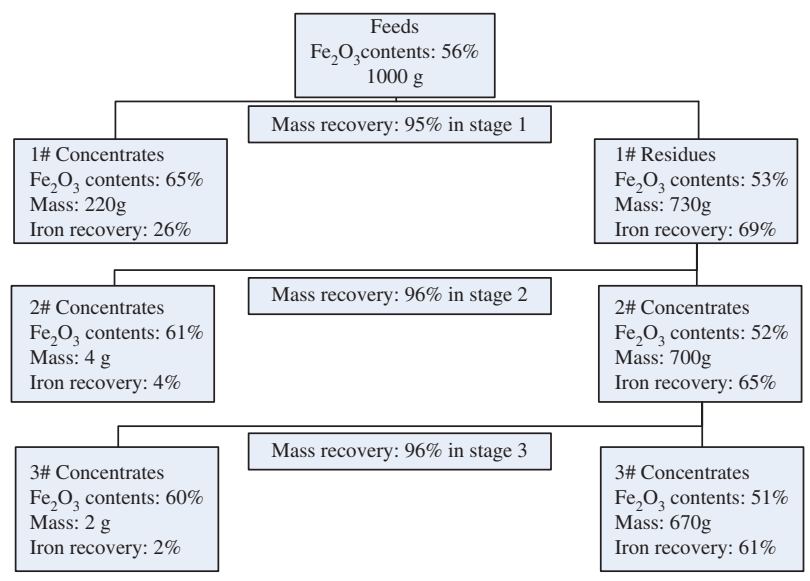

Figure 5. Iron contents of the RM samples and the corresponding recoveries $(\mathrm{w} / \mathrm{w})$ in three magnetic separation stages. 
the second separation stage. In the second and third stages, 4 and $2 \mathrm{~g} \mathrm{RM}$ was captured in the steel wool matrix, respectively. The iron content of the concentrates in the second and third separation stages was decreased to $61 \%$ and $60 \%$, respectively. After three separation stages, the raw RM was separated into $69 \%(\mathrm{w} / \mathrm{w})$ residue fractions and $31 \%(\mathrm{w} / \mathrm{w})$ concentrate fractions. Iron content in the RM concentrates and residues was $64 \%$ and $51 \%$, respectively. There was about $32 \%$ iron in raw RM collected in the high iron RM after three stages magnetic separation.

The processing capacity of RM in dry weight could be higher than 10 ton/h by using the circulating superconducting magnetic separator. Hematite was the main iron mineral phases in the RM. The RM with high iron content could be used in the blast furnace iron making process. The superconducting magnetic separator was energy saving and of large processing capacity, which could be practical in reduction of the disposal RM.

It was reported in the previous work,[7] a reciprocating superconducting magnetic separator was used in iron recovery from RM. The grade of the iron-rich RM concentrate was increased from $59 \%$ to $68 \%$ when the magnetic field was $2 \mathrm{~T}$. The mass recovery of the RM concentrates was about $10 \%$. The magnetic filtration path of the reciprocating superconducting magnetic separator was about $1000 \mathrm{~mm}$. Particles captured in the steel wool matrix was hard to be washed out in the long filtration path. In this work, the filtration path of the circulating box was $100 \mathrm{~mm}$. The physical retention of RM particles in the steel wool matrix was weakened. The short filtration path of the circulating magnetic separation system was advantaged for the RM treatment. At the same time, the circulating boxes are easy for maintenance and replacement.

\subsection{Effects of steel wool}

Figure 6 showed the iron oxide contents and recoveries of the RM concentrates and residues after the magnetic separation. As shown in Table 3, the order of the magnetic-field gradient produced by steel wool was $2 \#>3 \#>4 \#>5 \#$. The grade of the iron-rich RM concentrates decreased when finer steel wool was filled in the circulating boxes. It is well known that larger magnetic field and magnetic-field gradient provide greater magnetic drag force of the magnetic particles. It was shown that simply increasing the magnetic drag force could decrease iron content in the RM concentrates. The results were consistent with previous work that larger magnetic field decreased iron content in the RM concentrates when the magnetic-field gradient was maintained.

The mass recovery of the RM concentrates was higher than $20 \%$ when $3 \#$ steel wool was used. The iron content in the RM concentrates was higher than $63 \%$, which was $8 \%$ increase compared with the raw RM. The mass recovery of the RM concentrates decreased to less than $14 \%$ when $5 \#$ steel wool was used. However, the grade of the iron-rich RM concentrate was increased to $68 \%$ when $5 \#$ steel wool was filled. The parameters of the steel wool matrix should be selected in such a way that the recovery and the grade are optimized simultaneously.

The mass recovery and iron content of the RM residues after magnetic separation are shown in Figure 6(b). The iron content and mass recovery of the RM residues increased with finer steel wool used. The iron recovery in the magnetic separation process decreased from $46.4 \%$ to $15.6 \%$ when $5 \#$ steel wool was filled instead of 2\#. Greater magnetic drag force in the magnetic separation process led to higher iron recovery in the RM concentrates.

In previous works, steel wools were compacted in moulds before filled in the box.[7] It was found that RM was hard to be washed out in the concentrates collecting process. Therefore, in this work, 150,250 and $500 \mathrm{~g}$ steel
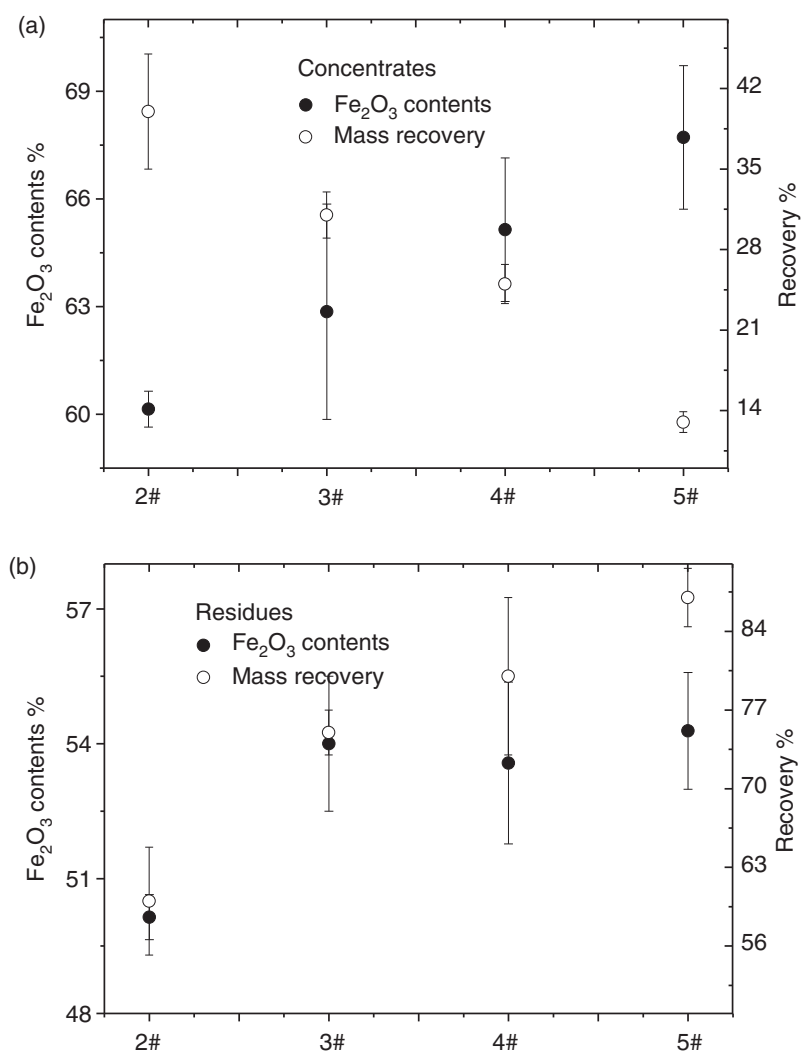

Figure 6. Iron contents and recoveries in the RM samples with different steel wools used.

Table 4. Effects of steel wool packing density on the iron recovery from $\mathrm{RM}$.

\begin{tabular}{lccc}
\hline $\begin{array}{l}\text { Packing } \\
\text { density }\end{array}$ & $\begin{array}{c}\text { Iron content } \\
\text { in concentrates }\end{array}$ & $\begin{array}{c}\text { Recovery of } \\
\text { concentrates }\end{array}$ & $\begin{array}{c}\text { Iron content } \\
\text { in residues }\end{array}$ \\
\hline 150 g per box & $66.3 \pm 1.9$ & $12.3 \pm 1.2$ & $54.3 \pm 2.0$ \\
250 g per box & $64.3 \pm 1.7$ & $17.9 \pm 1.8$ & $53.4 \pm 2.4$ \\
500 g per box & $65.1 \pm 2.0$ & $25.0 \pm 1.7$ & $53.6 \pm 1.8$ \\
\hline
\end{tabular}


Table 5. Particle size and Impurities of the RM samples.

\begin{tabular}{lcc}
\hline RM Samples & Particle size $\mathrm{D}(4,3) \mu \mathrm{m}$ & Opaque material $\%^{\mathrm{a}}$ \\
\hline Raw RM & 7.49 & 10.35 \\
Concentrate RM & 8.72 & 15.52 \\
Residue RM & 6.24 & 7.77 \\
\hline
\end{tabular}

${ }^{a}$ Quantitative analysis of the opaque materials in the micrograph of RM.

wool were filled in the box without mould compacting. The effects of packing density on the magnetic separation are shown in Table 4. Iron contents in the RM concentrates were not affected by the packing density. The recovery of RM concentrates decreased with less steel wool filled in the box.

The particle size distributions of RM in HGMS with steel wool filled were investigated in the previous work.[7] Physical entrapment of large RM particles $(>100 \mu \mathrm{m})$ in the steel wool matrix was observed in the magnetic separation. The magnetic aggregation of fine RM in superconducting magnetic field may be important to the magnetic separation. The interactions between fine RM particles in the steel wool matrix should affect the recovery of iron-rich RM particles.

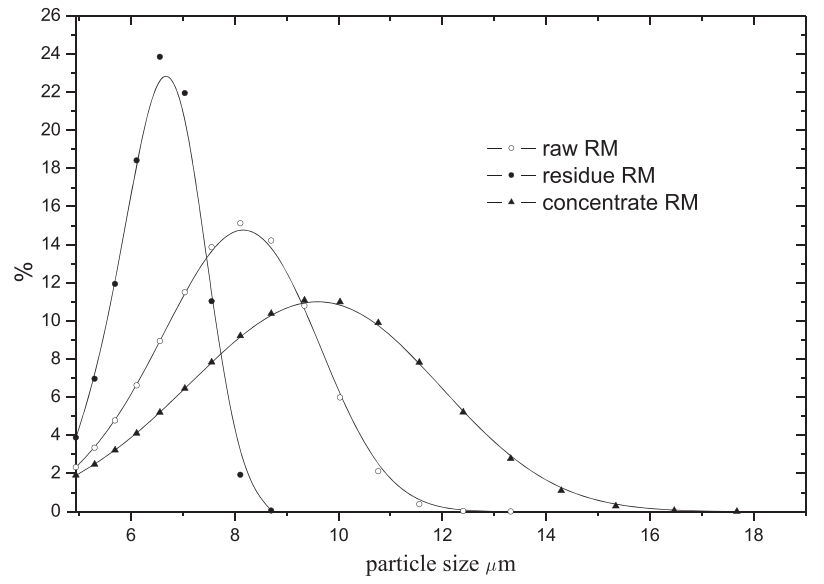

Figure 7. Particle size distributions of the raw, concentrate and residue RM. The proportions of large particles in the concentrate RM increased compared with raw and residue RM.

Iron-rich RM captured in the pores of the steel wool matrix may block the particles' movement, therefore, increases the concentrates recovery and decreases the iron content in concentrates. The movement path of RM particles in steel wool matrix of this work was $100 \mathrm{~mm}$, which was much shorter (a)

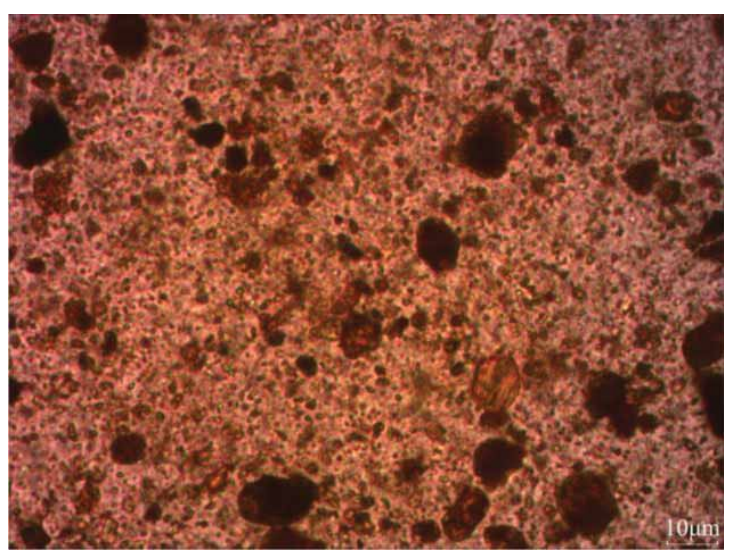

(b)

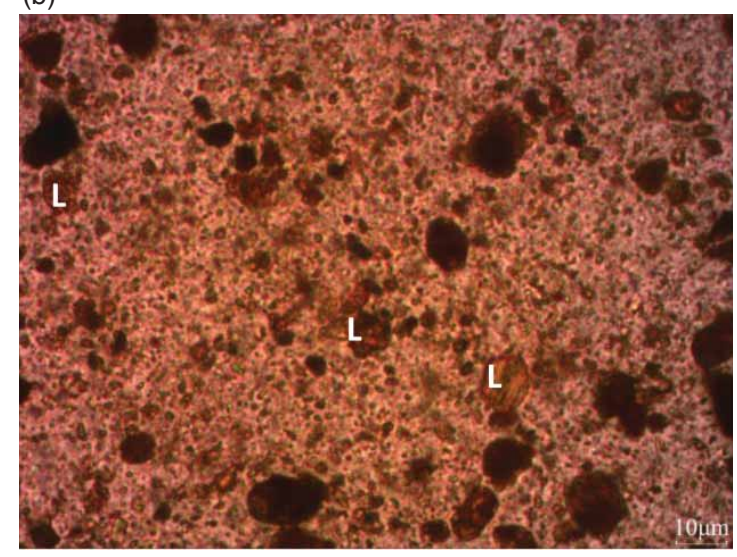

(c)

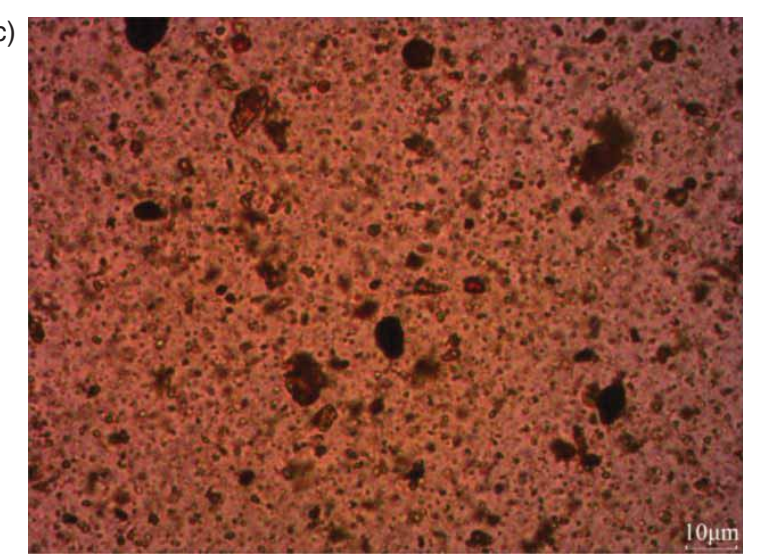

Figure 8. Micrograph and microanalysis of the raw (a), concentrate (b) and residue RM (c). Opaque proportions of the RM samples were shown in the photographs. 
than previous work $(1000 \mathrm{~mm})$.[7] This may be one reason for the higher iron content in the RM concentrates in this work.

\subsection{Particle size distributions}

The particle size distributions of the raw, concentrate and residue RM are shown in Figure 7. The mean particle size $\mathrm{D}(4,3)$ of the feed, concentrate and residue RM is shown in Table 5. The mean particle size $\mathrm{D}(4,3)$ of $\mathrm{RM}$ increased from 7.49 to $8.72 \mu \mathrm{m}$ after the magnetic separation. Large particles of RM could be physically entrapped in the micropore of the steel wool matrix. In this way, large particles with or without magnetic component could be collected in the concentrate RM. Therefore, iron oxide content of the concentrate RM was decreased for the physical entrapment in steel wool matrix.

\subsection{Micrograph and microanalysis of RM}

The micrograph and microanalysis of the raw, concentrate and residue RM are shown in Figure 8. Large opaque particles enriched in the concentrated RM compared with Figure $8(\mathrm{a})$ and $8(\mathrm{~b})$. The changes of particle size distributions shown in the photomicrograph were consistent with the laser particle size analysis. It is well known that hematite is opaque proportions, while quartz, calcite and $\mathrm{Al}(\mathrm{OH})_{3}$ are light-transmissive components. Large lighttransmissive particles such as 'L' particles in Figure 8(b) were observed. It could be speculated that large nonmagnetic particles were entrapped in the steel wool matrix and then accumulated in the concentrated RM, the quantitative analysis of the dark proportions in raw, concentrate or residue RM is shown in Table 5. The variations of dark proportion contents in the photomicrograph were consistent with the changes of iron oxide in the raw, concentrate and residue RM after magnetic separation.

\section{Conclusions}

A circulating high-gradient superconducting magnetic separator was produced for RM treatment in this work. Circulating boxes filled with steel wool was easy to wash and maintain. The filtration path length of $100 \mathrm{~mm}$ in circulating box could reduce the physical retentions. The raw RM sample with $56 \%$ iron content was fed in the magnetic separation system. The RM concentrates of $65 \%$ iron contents were collected after magnetic separation. The mass recovery of the RM concentrates was $25 \%$. Large magnetic-field gradient increased the iron recovery ratio, but decreased the iron content of the RM concentrates.

\section{Acknowledgement}

We gratefully acknowledge Ting Wang, Xiaotao Wei and Bo Gong (Qihuan Mechanical \& Electronic Engineering Corp.) in operating the superconducting magnets.

\section{Funding}

Financial support was provided by Ph.D. program's foundation of East China Institute of Technology, National Natural Science Foundation of China [No. 21207017], National Natural Science Foundation of Jiangxi province [No. 20122BAB216001] and Scientific Research Fund of Jiangxi Provincial Education Department [No. GJJ13437].

\section{References}

[1] Sushil S, Batra V. Catalytic applications of red mud, an aluminium industry waste: a review. Appl Catal B: Environ. 2008;81:64-77.

[2] Bhatnagar A, Vilar VJP, Botelho CMS, Boaventura RAR. A review of the use of red mud as adsorbent for the removal of toxic pollutants from water and wastewater. Environ Technol. 2011;32:231-249.

[3] Snars K, Gilkes RJ. Evaluation of bauxite residues (red muds) of different origins for environmental applications. Appl Clay Sci. 2009;46:13-20.

[4] Liu W, Yang J, Xiao B. Application of Bayer red mud for iron recovery and building material production from alumosilicate residues. J Hazard Mater. 2009;161:474 478.

[5] Liu W, Yang J, Xiao B. Review on treatment and utilization of bauxite residues in China. Int J Miner Process. 2009;93:220 231.

[6] Svoboda J. Recent developments in magnetic methods of material separation. Miner Eng. 2003;16:785-792.

[7] Li Y, Wang J, Wang X, Wang B, Luan Z. Feasibility study of iron mineral separation from red mud by high gradient superconducting magnetic separation. Phys C: Superconductivity. 2011;471:91-96.

[8] Li Wd. Study on the new ore dressing of Bayer red mud [Master thesis]. Central South University; 2005.

[9] Ohara T, Kumakura H, Wada H. Magnetic separation using superconducting magnets. Phys C: Supercond. 2001;351:1272-1280.

[10] Newns A, Pascoe RD. Influence of path length and slurry velocity on the removal of iron from kaolin using a high gradient magnetic separator. Miner Eng. 2002;15:465-467.

[11] Nakai Y, Mishima F, Akiyama Y, Nishijima S. Development of high gradient magnetic separation system under dry condition. In Physica C: Supercond. 2010;470:1812-1817.

[12] Mishima F, Terada T, Akiyama Y, Nishijima S. High gradient superconducting magnetic separation for iron removal from the glass polishing waste. Appl Supercond, IEEE Trans. 2011;99:2059-2062.

[13] Nishimura K, Miura O, Ito D, Tsunasima Y, Wada Y. Removal of radioactive heavy metal ions from solution by superconducting high-gradient magnetic separation with schwertmannite and zirconium-ferrite adsorbents. IEEE Trans Appl Supercond. 2009;19:2162-2164.

[14] Ghosh I, Guha S, Balasubramaniam R, Kumar AVR. Leaching of metals from fresh and sintered red mud. J Hazard Mater. 2011;185:662-668. 